\title{
Le voyageur sans bagage ou l'épreuve du récit des mobilités : réflexivité de l'enquêté et méthode de cadrage
}

\author{
The traveler without luggage or proof story of mobility: \\ reflexivity of the respondent and framing method
}

\section{Denis Martouzet}

Reçu le 2 novembre 2013; accepté le 2 juin 2014

(C) IFSTTAR et Éditions NecPlus 2014

\begin{abstract}
Résumé Une pièce de théâtre de Jean Anouilh, Le voyageur sans bagage, illustre la situation d'un individu amené à se confronter à son propre passé, comme l'est l'enquêté face à un enquêteur sollicitant un récit de vie. Un rejet partiel de ce passé est toujours possible : la personne met en œuvre des stratégies pour dire et se montrer à soi-même un passé acceptable. Après examen de certaines de ces stratégies, nous proposons une méthode de captation qui permet de réduire les conséquences des biais qui ne manquent pas d'apparaître en situation d'enquête, dans le cas d'un récit de mobilité, récit de vie précisément orienté sur les mobilités de l'individu à l'échelle biographique, à partir d'un programme de recherche mené pour le compte du puca portant sur les valeurs mobilisées dans les discours de justification de la mobilité d'un échantillon de personnes.
\end{abstract}

Mots clés mobilité · méthodologie - enquête qualitative · enquête quantitative · rationalisation · réflexivité

\begin{abstract}
The play by Jean Anouilh, The traveler without luggage, illustrates the situation of an individual who is confronted with his own past, as is the defendant facing an investigator who is seeking a life story. Partial rejection of the past is always possible: the person implements strategies to tell and show himself a more acceptable past. After reviewing some of these strategies, we propose a method of recording that can reduce the effects of bias which appear in inquiry situations, in the case of narrative mobility (storytelling which is oriented to mobility), based on a research program conducted on behalf of PUCA (Plan Urbanisme Construction Architecture), concerning values
\end{abstract}

Denis Martouzet $(\triangle)$

Université de Tours, Équipe IPAPE - UMR 7324 CITERES, 35 allée Ferdinand de Lesseps

e-mail : denis.martouzet@univ-tours.fr expressed by a cross-section of people in their discourse justifying mobility.

Keywords mobility - methodology - qualitative research · quantitative research $\cdot$ rationalization $\cdot$ reflexivity

Le voyageur sans bagage est une pièce de théâtre écrite par Jean Anouilh, jouée pour la première fois le 16 février 1937. Elle est classée, par l'auteur lui-même, dans les pièces noires. Le thème en est sombre bien que le traitement soit ponctué de moments d'humour ou de légèreté qui, par contrepoint, assombrissent encore l'ensemble. Ce thème, le poids du passé, est exploré à travers l'histoire d'un homme encore jeune frappé d'amnésie depuis l'épreuve du front en 1918 et qui, après une vingtaine d'années dans un asile, est retrouvé, parmi beaucoup d'autres, par sa famille, du moins le suppose-t-on puisque le déroulement de la pièce n'indique que très tardivement si cette famille est bien la sienne. Les membres de cette famille cherchent à lui rappeler son passé, en lui contant plusieurs anecdotes de son enfance, de son adolescence, de son départ à la guerre. Si la mémoire ne lui revient pas vraiment, les indices sont troublants et il a alors la crainte d'être celui qu'on lui décrit. Il est vrai que les souvenirs qu'on lui attribue ne sont guère reluisants, sans que pour autant ce soient ceux d'un criminel, juste ceux d'un égoïste un peu méchant. «Je ne suis pas Jacques Renaud; je ne reconnais rien ici de ce qui a été à lui [...]. Voyez-vous, pour un homme sans mémoire, un passé tout entier, c'est trop lourd à endosser en une seule fois. Si vous vouliez me faire plaisir, pas seulement me faire plaisir, me faire du bien, vous me permettriez de retourner à l'asile. » Il ajoutera plus loin, de façon concise : « je suis lavé de ma jeunesse » et quand sa belle-sœur, face à l'évidence du manque d'effort qu'il fait pour recouvrer la mémoire ou au moins se souvenir d'un fait ou deux, lui assène : «Alors, vous ne retrouverez jamais votre mémoire », il conclut qu'il « en arrive à le souhaiter. » 
Ce texte pose le postulat que le passé est lourd à porter, peut-être trop lourd en certaines circonstances ou pour certaines personnes. Cependant, pour montrer cela et notamment le fait de l'omniprésence du poids de ce passé, nous ne mobilisons pas des récits de parcours de vie difficile, dont certains éléments nécessitent la remémoration d'épisodes douloureux et traumatisants, comme le présente Pierre Bourdieu dans La misère du monde [1] par exemple. $\mathrm{Au}$ contraire, nous mettons l'accent sur des situations banales, quotidiennes, qui ne renvoient pas à de terribles et lourds secrets familiaux ou personnels et nous montrons, a contrario, que ce type de situation ne survient pas que dans des récits de vie mais aussi dans des enquêtes portant sur le court terme, le quotidien, le récent. Nous servant de cette pièce comme prétexte à interrogation, nous prenons au sérieux ce postulat d'un passé lourd à porter, examinant en quoi la situation d'enquête peut justement mettre la personne qui subit un entretien ouvert en situation de devoir se confronter à son passé de façon plus directe et immédiate qu'en temps normal, de devoir faire avec celui-ci, quels que soient les événements qui ont composé ce passé. Nous postulons aussi que ceci n'est pas anodin. Bien évidemment, des différences existent entre la situation de l'enquêté et celle de ce Jacques Renaud. Le passé de celui-ci lui revient presque d'un bloc, puisque sa mémoire est, au départ, vierge et que son passé lui revient en l'espace de quelques heures, le temps de la pièce de théâtre, tandis que l'enquêté l'a toujours en partie présent à l'esprit, et qu'il ne fait que le remobiliser : l'écart entre un avant et un après est infiniment moins grand, mais il existe. Les cinq tableaux proposés par Jean Anouilh apparaissent alors comme un raccourci qui met en évidence l'importance de la remémoration et de ses conséquences : se souvenir de ce que l'on avait cru avoir pu oublier. D'autre part, le personnage d'Anouilh parvient à rester à distance de son passé : il est amnésique et, à mesure que l'on avance, il joue à l'amnésique et est en situation, à la fin de la pièce, de pouvoir accepter ou refuser le passé qui s'avère être le sien. Une personne enquêtée peut-elle aussi avoir des stratégies permettant de jouer avec son passé, y compris à très court terme, d'en accepter une partie, une autre non? L'analyse de certains comportements discursifs et cognitifs semble permettre une réponse positive à cette question et, ainsi, d'envisager des possibilités de réécriture du passé.

Cet article se veut d'abord méthodologique. Il mobilise, néanmoins, nécessairement, des éléments empiriques : ceux-ci permettent d'illustrer ou de conforter les aspects méthodologiques mis en avant. L'objectif majeur de cet article, au-delà de la présentation d'une méthode visant à appréhender la signification de la mobilité des individus dans leur quotidien, est l'évaluation de cette méthode. Elle propose le couplage d'une mesure quantitative des déplacements, par le truchement de loggers-GPS ${ }^{1}$, et d'une saisie qualitative, par entretiens, de la signification que les individus donnent à leur mobilité. On sait les avantages et les limites de cet appareillage GPS [2;3;4;5]. Nous en présentons ici un aspect méthodologique fort : le protocole mis en œuvre a permis de réduire de manière significative certains des biais de recueil d'information, biais liés à la relation que la personne entretient avec son passé et avec elle-même.

Nous abordons ainsi, dans un premier temps, la question de l'enquête avec pour angle d'analyse les statuts qu'il est possible de conférer au passé et les biais que cela peut contribuer à faire émerger. Ensuite, nous proposons la restitution du mode opératoire mis en œuvre dans une recherche, intitulée Périvia, le périurbain à l'épreuve $d u$ durable, menée pour le compte du Plan urbanisme construction architecture, dans le but de montrer une possible technique de réduction de ces biais [6].

\section{Passé, enquête et gestion présente du passé en situation d'enquête}

\subsection{Les statuts du passé et la situation d'enquête}

Comme en de multiples autres situations, il s'agit, dans les techniques relevant des entretiens dans le cadre des méthodes relatives aux sciences humaines et sociales, quelle que soit la forme de ces techniques, de recueillir des éléments relatifs à une personne, quant à ce qu'elle est ou n'est pas, ce qu'elle a été ou non, ce qu'elle pense être ou n'être pas (ou avoir été, faire ou avoir fait, pensé ou avoir pensé... ou non), par le discours qui repose tant sur ce qui relève de la logique que de la rhétorique. Ce discours nécessite indubitablement la remémoration et la mobilisation d'éléments de natures diverses (souvenirs mais aussi modes argumentatifs). Selon la posture de recherche, en lien avec le type de résultats attendus, il peut s'agir de saisir ce qui relève du témoignage et, dans ce cas, la question de la réalité, plus précisément celle du rapport de l'individu au réel est centrale, ou bien de capter la signification, au sens large, que donne l'individu à ce réel ou, plus précisément, à la représentation qu'il s'en fait. Par ailleurs, dans une posture pragmatiste, ce n'est pas tant le rapport au réel qui est visé que la compréhension des causes qui ont amené l'individu, à un moment donné dans la situation d'enquête,

\footnotetext{
${ }^{1}$ Un logger-GPS est un petit boitier (environ $2 \mathrm{~cm} \mathrm{x} 2 \mathrm{~cm} \times 1 \mathrm{~cm}$ ) permettant d'enregistrer, selon un pas de temps qu'il est possible de programmer, sa position géographique précise. Il est ainsi possible de suivre précisément les cheminements d'une personne qui le porte sur elle.
} 
à se sentir obligé de dire ce qu'il a dit. Quelle que soit la posture et les modalités pratiques d'investigation, dans la plupart des discours, le passé, nécessairement mobilisé, a plusieurs statuts ou fonctions. Il a, si on suppose un niveau minimal de capacité à l'objectivité chez l'individu - en fait si on lui récuse la possibilité de pure subjectivité - , la fonction de témoignage, que ce soit le témoignage de l'interviewé sur sa propre personne, sur sa famille, sur une situation, un groupe, une époque, un lieu, un fonctionnement, un événement, une profession, une société, etc. ${ }^{2}$ Deuxièmement, ce passé a aussi valeur explicative, par l'enchaînement des relations de cause à effet : dire ce qui a été implique de dire pourquoi cela a été et les raisons de ce pourquoi - présentées comme des causes - sont, pour beaucoup, mobilisées dans le passé antérieur à l'élément décrit. Ce faisant, ce passé a un sens, en tout cas lui est donné un sens puisque, justement, il est mobilisé soit comme témoignage descriptif, c'est-à-dire comme élément choisi parmi d'autres puisque tout ne peut être dit, soit comme explication.

Par ailleurs, cette signification donnée intentionnellement n'épuise pas toute la signification de ce qui est dit : dire quelque chose dit plus que ce qui est, au sens strict, dit. Nous pouvons illustrer cela par l'une des plus célèbres phrases de Claude Lévi-Strauss [7], célèbre justement non par ce qu'elle dit mais par ce que, dans le non-dit, elle implique : « Le village entier partit le lendemain dans une trentaine de pirogues, nous laissant seuls avec les femmes et les enfants dans les maisons abandonnées. » Il y a bien valeur de témoignage, une certaine objectivité quant au fait relaté et il y a, parallèlement choix parmi tout ce qui aurait pu être écrit (cet élément de description a, sans doute, plus d'importance que d'autres aux yeux de l'ethnologue, dans cet ouvrage où le chercheur se livre). Il y a bien aussi, au moins de façon implicite, une signification explicative (c'est parce que lui et ses compagnons, sont restés seuls au village avec les femmes qu'il a pu, ensuite, en faire une certaine analyse : le fait d'être seul n'est pas simplement une circonstance, c'est un véritable contexte). Mais ce n'est pas parce qu'existent ces significations qu'il n'en existe pas d'autres, qui échappent à celui-là même qui parle (ou écrit), à son insu. Ainsi, par exemple, cette phrase telle qu'elle est formulée révèle la place que l'auteur confère à la femme, qui n'est rien puisque tout le village est parti. On peut argumenter que Lévi-Strauss n'est alors que le reflet d'une société et d'une époque - les siennes - qui mésestiment le genre féminin. Toujours est-il que, disant cela, il en dit plus que ce qui est simplement énoncé, par les implications logiques de ce qui est énoncé. Mais

\footnotetext{
${ }^{2}$ Le témoignage n'implique pas pure objectivité mais nécessite la possibilité d'une certaine objectivité.
}

au-delà de cet exemple nécessairement particulier, toute personne qui parle sait, même si elle l'oublie souvent, que l'ensemble des significations dépasse largement le cadre de la signification voulue par le locuteur dans la situation d'échange. Le langage nous échappe toujours en partie et, parce que nous savons cela, nous mettons systématiquement en œuvre, de façon plus ou moins efficace, des stratégies de réduction du sens que prend, au-delà de celui que l'on voudrait strictement conférer à nos dires, ce que nous disons.

La situation d'entretien se caractérise notamment par le fait que le présent du discours et le passé (avec d'autres éléments) contenus dans le discours sont inextricables : le passé, en tant qu'il est relaté à autrui (voire à soi-même) n'a de sens que dans le présent du discours qui lui-même ne peut s'expliquer que par le passé en tant qu'il a valeur explicative du présent. Il y a reconstruction, c'est-à-dire que, en tant que re-construction, le discours n'a pas forcément beaucoup à voir avec ce qui est advenu, ni même avec ce que le locuteur croit être advenu, mais en tant que construction il ne peut pas être radicalement différent de ce qui est advenu ou de ce que le locuteur croit être advenu. Comme il y a reconstruction, il y a un jeu (au sens où l'on dit qu'il y $\mathrm{du}$ jeu dans une mécanique mais aussi par le fait que le locuteur joue) que le locuteur utilise, volontairement ou non, consciemment ou non, pour ne pas tout dire, de façon à donner une certaine image de soi à l'enquêteur, de façon à lui plaire, lui faire plaisir en donnant les bonnes réponses... Ces biais d'enquête ont largement été décrits et analysés $[8,9,10]$.

Par ailleurs, par le fait même que, avant reconstruction, il y a mobilisation d'éléments (parmi lesquels est fait un tri) et pour cela il y a remémoration, plus ou moins instantanée selon les éléments remémorés, la situation de l'enquêté est semblable à celle de Jacques Renaud, ce voyageur qui a toute la légèreté de celui qui ne traîne pas de bagage, toutes proportions gardées : la personne enquêtée, ne partant pas d'une mémoire vierge, ne reçoit pas tout son passé d'un coup, mais la situation d'enquête l'amène à avoir à l'esprit plus de passé qu'en dehors de la situation d'enquête ou pas le même passé qu'en temps normal. Non qu'il ait plus de bagages - son passé est identique qu'il le dise et le veuille ou non - mais il a alors plus conscience du poids de ces/ses bagages.

Un entretien - comme nombre d'autres types de situations - remet en question le rapport que chacun entretient avec la réalité, avec l'autre, avec soi. De là, et notamment du fait de la remise en question du rapport à soi, la stabilité ontologique de la personne est possiblement mise à mal par cette situation d'enquête. Le simple fait d'énoncer une action réalisée par soi dans le passé conduit à la requestionner et, donc, à se requestionner quant au bien-fondé de cette action : c'est toujours une remise en question, déstabilisant la personne plus ou moins 
durablement. C'est, par exemple, le cas, observé dans les entretiens menés pour la recherche Périvia, de la personne qui, parce qu'elle est amenée à dire certaines de ses pratiques de mobilité, s'aperçoit elle-même que celles-ci ne sont pas respectueuses de valeurs, de normes auxquelles elle adhère ou est censée adhérer. Ce qui compte n'est pas tant le possible jugement de valeur de l'autre, de l'enquêteur, ni la culpabilisation qui s'ensuivrait, que le regard de l'enquêté sur lui-même. C'est la découverte de sa duplicité : elle ne fait pas (exactement) ce qu'elle doit faire, elle ne fait pas ce qu'elle croit faire. Non seulement elle découvre ce décalage mais aussi en même temps le fait qu'elle connaissait ce décalage. Il y a duplicité et duplicité quant à sa propre duplicité.

Pour préserver cette stabilité, la personne enquêtée met en œuvre des stratégies qui lui permettent effectivement de la sauvegarder en situation d'entretien. Cependant, finalement, l'enquête une fois passée pourra la mettre encore plus à mal : son passé, dans le futur, contiendra cet entretien qui l'a amenée à être en rupture puisqu'elle a mis en œuvre - et elle le sait - des stratégies inhabituelles.

Enfin, plus largement, il est possible d'analyser, au-delà du contenu même des dires recueillis lors d'un entretien de type récit de mobilité, une situation d'enquête par trois entrées : le rapport que l'interviewé entretient avec la réalité, celle-ci étant nécessairement, mais pas uniquement, une réalité passée, le rapport entretenu avec l'autre, c'est-à-dire l'enquêteur, celui à qui est dit cette réalité, enfin le rapport à soi-même, c'est-à-dire l'ambivalence, chez une même personne, entre celui qui parle - et ne dit pas tout - et celui qui s'entend parler - et n'entend donc pas tout.

Au final, toute situation d'enquête s'avère être, au moins pour l'enquêté, la confrontation du passé et du présent à l'image même de ce qu'avait écrit Saint Augustin [11] sur la question du temps et pour qui «on ne peut dire, à proprement parler, qu'il y ait trois temps, le passé, le présent et le futur ; mais peut-être serait-il plus juste de dire : "Il y a trois temps, le présent des choses passées, le présent des choses présentes, le présent des choses futures" ». Il donne plusieurs exemples, dont celui-ci : «Mon enfance, par exemple, qui n'est déjà plus », écrit-il dans le livre 11 des Confessions, « est dans le passé, qui lui-même n'est plus ; mais son image, lorsque j'évoque son souvenir et que j'en parle aux autres, c'est dans le présent que je la vois, parce qu'elle est encore dans ma mémoire ». Cependant, il ne qualifie pas le passage de l'événement passé au souvenir qui en reste et qui est nécessairement autre et le décalage entre la réalité pleine et entière et ce que, le temps aidant, l'on en fait.

Cela signifie par ailleurs que, de ce point de vue, la situation d'enquête n'est pas fondamentalement différente des situations ordinaires et que la situation de Jacques Renaud n'est extraordinaire que par le raccourci temporel que permet l'amnésie. Il n'y a pas seulement souvenir présent du passé, il y a gestion de ce souvenir.

\subsection{La gestion présente du passé}

Le passé est, une fois pour toutes. Il est figé, fixé, inchangeable. On n'a pas d'autres choix que de devoir faire avec ce passé. D'un autre côté, le rapport que l'on a avec ce passé, lui, est modifiable, il est même en constante évolution. Il existe une capacité d'oubli, plus ou moins importante, plus ou moins orientée. Il existe aussi une capacité d'insouciance qui permet que soit jugé de peu d'importance tel événement passé sans que pour autant il soit oublié. Par ailleurs, même si l'on admet, dans une optique freudienne, que le passé est entièrement intégré au présent de l'individu, sous le mode du souvenir ou celui du refoulement (« les souvenirs oubliés ne sont pas perdus » nous dit Freud [12], dans sa deuxième leçon), le passé d'une personne n'est pas connu - ou si peu - des autres, notamment en ce qui concerne, plus que les faits et les actes, les raisons profondes de ces actes, les intentions qui y ont présidé, les intentions non suivies d'effets. .. Aussi, dans la relation d'enquête peut-on se donner une certaine liberté, dans les dires, relativement aux réalités passées.

L'expression faire avec renvoie à cette souplesse. Elle contient une double dimension. La première est celle d'une fatalité dans le sens où, le passé (ou le présent) étant ce qu'il est, on ne peut pas ne pas faire avec lui : il est une donnée première. La seconde dimension renvoie au fait que faire est un verbe d'action : on peut, par exemple, choisir les éléments mobilisés, en dire, au présent, ce que l'on veut. Faire avec, c'est déjà faire. On peut jouer avec les éléments qui le composent, mais jouer suppose nécessairement des règles du jeu et on ne peut donc pas faire (et dire) n'importe quoi avec le passé. Par exemple, on ne peut se forcer à oublier, on ne peut se forcer à croire à ses propres mensonges [13], aussi crédibles aux yeux d'autrui soient-ils ${ }^{3}$.

Nous examinons ici deux cas de gestion du passé : le sophisme de l'amortissement et la mauvaise foi. Ce ne sont, bien sûr, pas les seuls qui peuvent intervenir pendant une enquête. D'autres existent aussi, outre le mensonge, le non-dit volontaire ou involontaire, les petits arrangements avec la réalité, etc. sous-tendus par la volonté

\footnotetext{
${ }^{3}$ Le terme de mensonge est délicat d'utilisation tant sa connotation morale dans certaines cultures et pour certains individus est forte. Mentir doit être compris ici comme le fait de dire quelque chose de faux sans que cela soit par erreur : il y a toujours une intention, même si celle-ci n'est pas toujours explicite pour l'auteur même de cet énoncé faux. Cela entraîne et renforce le fait qu'il n'y a pas, de la part de l'enquêteur ni du chercheur, une quelconque portée morale dans l'analyse et l'interprétation - et la restitution de celles-ci - des propos recueillis.
} 
de masquer tout ou partie de soi, associés avec les effets de rhétorique qu'ils mobilisent. Cependant, ceux sur lesquels nous voulons insister sont des post-rationalisations qui, si elles s'expriment, en situation d'enquête, dans ce qui est donné à l'autre, supposent d'abord - et c'est leur spécificité - un rapport à soi. Ils nécessitent aussi une réécriture du passé, une réécriture de son propre passé.

Robert Nozick [14] examine ce qu'il nomme le sophisme de l'amortissement ou sunk cost fallacy. Son objectif est de montrer le caractère limité de la rationalité humaine en situation. Il prend l'exemple d'une personne qui juge elle-même qu'elle ne va pas assez souvent au théâtre. Elle met alors en place une stratégie visant à l'obliger ou du moins à l'inciter à s'y rendre plus fréquemment. Pour cela elle prend un abonnement qui, parce qu'il est payant mais permet d'obtenir des réductions par rapport au tarif normal, l'engage à y aller suffisamment pour que le coût de l'abonnement soit amorti par le nombre de places ensuite achetées au prix réduit. La personne va donc voir plus de pièces de théâtre.

L'analyse est la suivante. Tout d'abord, cet abonnement ne la force en rien à aller au théâtre de façon plus assidue. En effet, le coût de l'abonnement est passé donc définitivement perdu. Si réellement elle ne souhaite pas aller au théâtre (ce qui est probable puisqu'elle est obligée de trouver une stratégie pour s'y forcer), mais qu'elle y va, se sentant obligée, alors elle ajoute au coût d'abonnement, le coût de la place achetée à tarif réduit et l'éventuel coût du déplaisir à y aller.

En fait, il suffit qu'elle croie être ainsi forcée. Elle n'est forcée que par elle-même, même si elle choisit une stratégie qui l'amène à utiliser un moyen qui lui est extérieur, l'abonnement, mais ce lien n'est que symbolique, c'est bien elle qui a initié ce moyen. Elle se force donc à faire quelque chose qu'elle n'a pas envie de faire.

Cette analyse en termes économiques ou en termes de rationalité suppose que la personne fait comme si elle pouvait modifier la valeur (y compris monétaire) du coût de l'abonnement alors que celui-ci appartient définitivement au passé : il est payé. Un abonnement ne s'amortit pas, plus exactement il est immédiatement amorti dès qu'acheté. Elle cherche donc à réécrire le passé, et à ses propres yeux, si la stratégie fonctionne (car malgré tout elle peut être efficace), elle parvient à cette réécriture. Au final, la personne réécrit son passé (elle nie le coût passé de l'abonnement comme si ce coût était présent) de manière à pouvoir justifier son présent qui consiste à aller au théâtre alors que cela lui coûte financièrement et en termes d'envie : elle se réécrit elle-même, au présent.

Il faut ajouter, curieusement, que, si malgré son abonnement, la personne ne va toujours pas au théâtre, elle reste rationnelle, bien qu'ayant fait une erreur d'appréciation de l'efficacité de la stratégie finalement non mise en œuvre.
Tandis qu'allant au théâtre, elle ne l'est pas. Davidson, dont les travaux montrent qu'au final, le sophisme de l'amortissement est un cas particulier de self-deception, estime que ce type de mise en œuvre de stratégie non rationnelle survient plus souvent que rarement [15]. De façon plus explicite, Sartre, de son côté, laisse penser que la mauvaise foi est la règle ou l'une des principales règles d'attitude de l'être humain, envers les autres mais surtout envers soi-même : « elle peut même être l'aspect normal de la vie pour un très grand nombre de personnes » [16].

Le sophisme de l'amortissement est un cas particulier de mauvaise foi ${ }^{4}$. La mauvaise foi est le concept décrit et analysé en termes philosophiques par Jean-Paul Sartre dans le chapitre éponyme de L'Être et le néant [12]. La mauvaise foi sartrienne n'est pas ce qu'en dit le langage commun, renvoyant plutôt à la figure du menteur percé à jour et qui ne veut pas le reconnaître. La mauvaise foi selon Sartre est la posture mentale de celui qui se ment à lui-même en toute bonne foi. Démontant le mécanisme d'apparition, d'entretien et de renforcement de cet état, le philosophe insiste notamment sur le fait que la personne sait quelque chose (notamment la concernant) et, en même temps, croit ce même quelque chose, c'est-à-dire qu'elle n'en est pas sûre. Son comportement va accentuer cette tension : croyant, il n'est pas sûr et n'étant pas sûr, il va chercher à accumuler les preuves (discursives, vestimentaires, comportementales, attitudinales...), qui ne sont en fait que des indices, des signes - et non des preuves ${ }^{5}$ - de ce qu'il est socialement, professionnellement ou sur d'autres plans, en surjouant son rôle par exemple. Cela se traduit, par exemple, dans les phénomènes d'hypercorrection fautive décrits par Bourdieu et Passeron [19]. Ce faisant, par le fait même de chercher des preuves de ce qui est, il prouve et se prouve qu'il n'en est pas sûr. Croyant renforcer sa certitude, il ne fait que renforcer son incertitude : s'il était véritablement certain de ce qu'il est, il n'éprouverait pas le besoin de preuves, ni même d'indices.

La mauvaise foi est non de l'ordre du rapport à l'autre mais du rapport à soi : la personne est l'un et l'autre, trompeur et trompé, enquêteur et enquêté. En même temps, la situation d'enquête, qui est surtout de l'ordre du rapport à l'autre, contient aussi cette relation à soi-même qui apparaît comme un filtre, s'ajoutant à ceux que la personne met en œuvre, plus ou moins volontairement et consciemment, visant à donner une certaine image d'elle-même à l'autre. Il faut bien noter cependant que cette volonté de donner une

\footnotetext{
${ }^{4}$ Nous n'entrons pas ici dans le détail des débats philosophiques, sémantiques et logiques entre Sartre [16], Elster [13], Dupuy [17], Davidson [15] et Engel [18] sur les subtiles distinctions à opérer entre la mauvaise foi sartrienne et la self-deception davidsonnienne.

${ }^{5}$ C'est ce qui conduit J.-P. Dupuy [17] à parler de théorie évidentialiste de la rationalité au sens anglais de evidence, traduit par signe.
} 
belle image à l'autre ressortit parfois aussi de la mauvaise foi : il s'agit bien de chercher à prouver ce que l'on est et non seulement le montrer sincèrement. La gestion de cette tension se manifeste dans le cadre présent de la situation d'enquête. Mais ce présent est double. D'une part, il correspond, grammaticalement parlant, au présent de généralité (je suis épicier, je suis garçon de café, exemples que prend Sartre) qui peut renvoyer à l'essence même de la personne telle qu'elle se pense. D'autre part, il a aussi valeur de présent ponctuel, en situation (la personne œuvre, ici et maintenant, en situation, comme garçon de café ou, dans la situation d'enquête, il relate comment en telles circonstances il œuvre). Le premier est ontologique, le second actuel. Le premier englobe le passé, le second le remet en cause tout en le confortant. La personne cherche, en même temps qu'elle écrit son présent par son action, à réécrire son passé.

Ces deux exemples de situation montrent la possibilité, parfois - ou souvent - utilisée, de réécrire le passé et, en situation d'enquête, d'ajouter un biais supplémentaire lorsque le protocole de recherche vise justement à mettre en évidence les justifications profondes de pratiques passées. Bien évidemment, la fonction principale de l'entretien est de recueillir ce que la personne veut bien dévoiler de sa personne, de ses pratiques, de ses pensées. Aussi, mettre en œuvre une méthode qui réduit ce biais revient à imposer un cadre qui permet d'atteindre un niveau de sincérité plus important ${ }^{6}$.

\section{Le cadrage du qualitatif par le quantitatif}

Tout entretien qualitatif renvoie aux biais évoqués précédemment et bien d'autres encore. De ce point de vue le chercheur ne peut envisager que deux postures, outre celle, non scientifique, de ne pas porter attention à cette question. Par ailleurs, la mise en œuvre du protocole de recherche situe le chercheur entre ces deux postures et non, idéalement, exclusivement sur l'une d'entre elle. Il peut, d'une part, considérer l'existence inévitable et nécessaire de ces biais et de leurs conséquences dans le contenu du matériau recueilli et prendre en compte voire d'en tirer parti dans la démarche d'analyse qui suit le recueil de matériau. D'autre part, il est possible de réduire, sans toutefois les faire disparaître, les biais en œuvre lors des entretiens ou minimiser l'impact de ceux-ci sur le matériau. Nous nous situons ici dans la posture qui tente d'amenuiser ces effets.

\footnotetext{
${ }^{6}$ Il convient de noter que cette sincérité est à nuancer puisque justement elle est cadrée. Cependant, dans ce qui suit, il faut garder à l'esprit que, indirectement, c'est la personne enquêtée elle-même qui fournit ce cadre.
}

\subsection{Le protocole de recherche : le couplage d'une mesure des déplacements et d'une justification discursive}

Une recherche pilotée par l'équipe IPAPE de l'UMR 7324 CITERES pour le compte du Plan Urbanisme Construction Architecture, menée entre 2009 et 2012, a visé la saisie de la signification que les personnes donnent à leurs déplacements. Plus précisément, il s'est agi de savoir si, parmi les facteurs intervenant dans les modalités de déplacement, des valeurs entraient dans la délibération amenant à choisir, pour un motif donné, tel mode de transport, telle destination, tel moment, tel parcours... telles et telles circonstances. Parmi les valeurs, terme qui est ici à prendre dans une acceptation large comme principe ayant capacité à orienter les modalités de décision et d'action, était tout particulièrement recherché celle de durabilité : est-ce que - et si oui comment ? Et combien ? - les principes contenus dans l'idée de durabilité entrent dans la délibération préalable à un déplacement ? Plus largement, est-ce que ces principes influencent les déplacements d'une personne et participent donc à la construction de sa mobilité en leur donnant sens?

À partir de ce questionnement, nous avons défini la mobilité d'un individu comme la signification que celui-ci donne à l'ensemble de ses déplacements, cet ensemble étant, de fait, la partie objective, observable et mesurable de sa mobilité $[20,21,22,23]$.

Il est clair que saisir l'ensemble des motivations profondes mais toujours en lien avec les aspects les plus triviaux des motifs de déplacements et des circonstances dans lesquelles ils peuvent se réaliser, et sous quelles contraintes, ne peut passer que par des entretiens ouverts et longs permettant de saisir les justifications que les personnes donnent à leurs déplacements. Cependant, encore faut-il connaître ceux-ci. Nous préférons le terme de justification à celui d'explication, pour trois raisons majeures. La première est pratique et méthodologique. Lors des entretiens menés, la question pourquoi? n'a jamais été posée : les pourquoi? peuvent être ressentis, plus ou moins fortement, comme une critique voire une accusation implicites biaisant les réponses fournies. Deuxièmement, a contrario d'une approche par justification, ce type de question et l'idée même d'explication renvoient à la raison, évacuant des réponses non rationnelles, renvoyant aux aspects les plus pratiques, circonstanciels, matériels, concrets. Le pourquoi a tendance à provoquer une réponse simple alors même que c'est très généralement un faisceau d'éléments qui, ensemble, permettent de comprendre la résultante qui est d'avoir agi ainsi et pas autrement. Chacun des éléments apportés n'a pas de valeur explicative suffisante. Troisièmement, en situation d'enquête et notamment dans la position de 


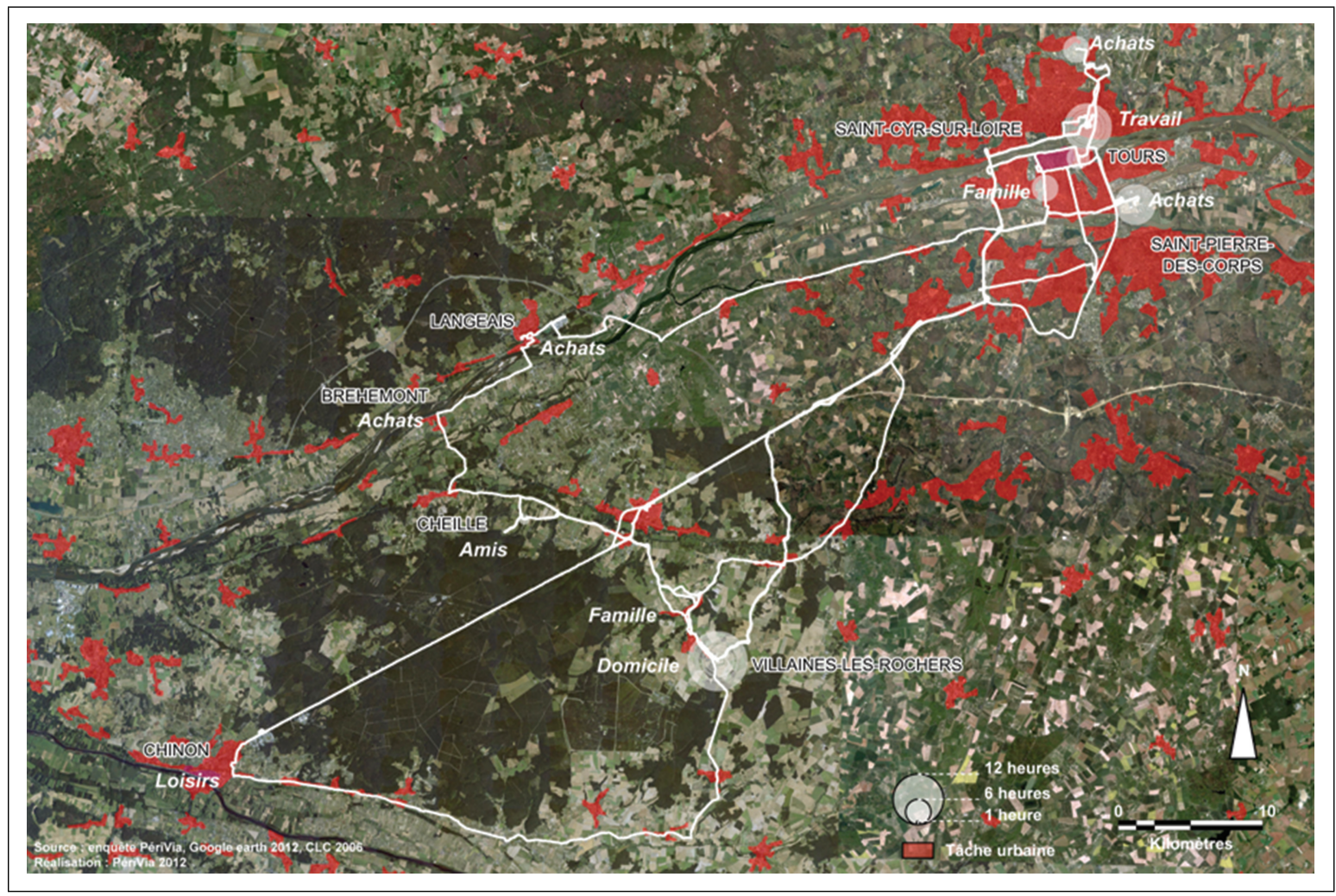

Fig. 1 Ensemble des déplacements d'une personne pendant une semaine ordinaire. Certains trajets peuvent avoir été effectués plusieurs fois, ce qui ne peut se distinguer ici. Lorsque la carte est présentée à son auteur, c'est-à-dire la personne ayant effectué ces déplacements, il y a possibilité de zoomer et d'afficher séparément et exclusivement les différents trajets réalisés à l'échelle d'une journée pour, ainsi, connaître le nombre de fois où tel chemin est suivi

l'enquêté sous le regard de l'autre - et d'autant plus si celui-ci pose des pourquoi ? explicites -, les explications fournies ne sont au final que des justifications aux yeux de l'autre et de soi-même : elles n'expliquent pas, elles fournissent une explication qui n'a pas toujours valeur explicative. Autant donc utiliser, de façon explicite, le terme de justifications puisque c'en sont. Cependant, ce terme est connoté péjorativement alors même que ce que nous cherchons n'est pas à évaluer en termes moraux. Par ailleurs, il renvoie directement aux biais liés à la situation de face-à-face (on se justifie auprès de l'autre) et ceux mis en avant précédemment dans le rapport que l'on entretient avec soi-même (on se justifie auprès de l'autre) ${ }^{7}$.

Pour répondre aux exigences de recueil de matériaux, nous avons mis en place le protocole suivant qui vise à garder l'idée de justifications en tant qu'elles sont les explications que la personne estime elle-même comme suffisantes pour légitimer son action, en ôtant le caractère péjoratif qui ne manque pas d'intervenir. Après le choix de l'échantillon de personnes en fonction des attentes

\footnotetext{
${ }^{7}$ Pour approfondir cette question, voir L. Thévenot [24].
}

liées à cette recherche ${ }^{8}$, nous avons équipé chacune de ces personnes d'un logger-GPS permettant de suivre l'intégralité de leurs déplacements pendant une semaine

\footnotetext{
${ }^{8}$ Plus précisément, l'échantillon retenu, qui n'a aucune prétention à une représentativité même relative, a privilégié la diversité des situations envisageables, en fonction notamment de la localisation du logement et du lieu de travail, de la profession exercée et des horaires qu'elle implique, de la composition de la famille, de l'âge, du genre et des ressources financières. Plus précisément, dans le cadre de l'agglomération de Tours et de ses couronnes périurbaines, 37 personnes ont été enquêtées. Elles ont un âge compris entre 14 et 74 ans (c'est-à-dire ayant une certaine autonomie dans leurs déplacements). Il y 22 femmes et 15 hommes. Ils représentent essentiellement les classes moyennes disposant de ressources leur permettant d'assurer, entre autres, leurs déplacements (19 professions intermédiaires ; 6 cadres et professions intellectuelles supérieures ; 5 retraités, 3 étudiants ; 2 artisans-commerçants, 2 employés). En termes de localisation du lieu de résidence, 16 personnes sont à moins de $10 \mathrm{~km}$ du centre de Tours, 10 à une distance de ce centre comprise entre 10 et $20 \mathrm{~km}, 6$ entre 20 et $30 \mathrm{~km}, 19$ entre 30 et 40,2 au-delà, jusqu'à $55 \mathrm{~km}$. Pour le couple lieu de travail/lieu de résidence, quatre catégories ont été distinguées : 20 individus habitent dans le périurbain et travaillent dans l'agglomération de Tours ; 11 individus habitent dans le périurbain et travaillent dans le périurbain ; 4 habitent l'agglomération de Tours et travaillent dans le périurbain ; enfin, 2 habitent dans l'agglomération de Tours et travaillent dans cette agglomération.
} 
que l'on a pu, avec elles, qualifier d'ordinaire, avec, sur le plan spatial, une précision métrique et, sur le plan temporel, une précision de l'ordre de la seconde. De là, nous avons pu en déduire la vitesse instantanée et, par l'analyse de la vitesse et du rythme de déplacement, en induire le mode de transport utilisé (à pied, en vélo, en deux-roues motorisés, en voiture, en transports en commun urbains, en train ou en TGV), confirmé par le tracé du trajet : les trajets recueillis ont été plaqués sur un fond de carte de type Google Earth $\AA$ (figure 1), ce qui a permis aussi de déterminer le motif principal du déplacement par la connaissance de la nature des points d'arrêts (entrée d'une école, station essence, cinéma, supermarché, club de sport...), confirmé par la durée d'arrêt : quelques minutes sur un parking de supermarché correspondent au débarquement ou à l'embarquement d'une personne ou encore à l'achat de carburant (mais la précision spatiale du tracé et du support cartographique permet de distinguer cela), une durée plus longue à un motif d'achat/loisirs (courses, shopping), une durée atteignant plusieurs heures renvoie au supermarché comme lieu de travail. Enfin, certaines activités ne nécessitent pas d'arrêt, comme une promenade en forêt, sont immédiatement repérables, par la vitesse et le rythme de déplacement. Lorsque la personne accepte le port du GPS, elle n'imagine pas, sans que pour autant cela lui soit caché, la somme et la précision des données qui peuvent être recueillies.

Le premier traitement de ces données est la synthèse sous forme d'une carte associée à un tableau (tableau 1) répertoriant chacun des déplacements et ses caractéristiques.

Le caractère technique de ces précisions a son importance dans la compréhension et la représentation que les personnes se font des résultats bruts puis retravaillés ainsi obtenus. Elles servent au cadrage de l'entretien.

\subsection{La phase d'entretien cadré}

Dans un second temps chaque individu ayant porté sur lui ce GPS passe un entretien long, composé de trois phases et ce n'est qu'au début de la troisième qu'est explicitement dit que l'objectif de la recherche est la saisie des valeurs présidant aux choix de déplacements et à la mobilité. La première phase vise l'établissement d'une sorte de carte d'identité mobilitaire, à partir du récit que donne la personne de sa vie dans ses grandes étapes (déménagement, changement d'institution scolaire, vie professionnelle...). Elle indique ainsi, par exemple, sa réticence à utiliser les transports en commun, la place qu'a eue le vélo tout au long de sa vie, l'importance de l'autonomie acquise par la maitrise des moyens de déplacements, l'impression de liberté associée à la voiture personnelle... Le questionnement porte là essentiellement sur des faits, non sur les justifications, encore moins sur les valeurs. Celles-ci transparaissent rapidement toutefois. La deuxième phase commence par la présentation à la personne de $s a$ carte, celle de ses trajets de la semaine avec GPS, carte que l'on peut zoomer quasiment à l'infini, associée au tableau détaillant à l'échelle de la semaine et pour chaque déplacement effectué les lieux de départ et d'arrivée, les distances parcourues et les vitesses moyennes, les modes de transport utilisés, les motifs de déplacement, les durées d'arrêt entre deux déplacements. La consigne donnée est la description de cette carte, globalement et jour après jour. L'ensemble des données étant trop important (la description pourrait durer des heures), l'enquêteur guide l'entretien mais les commentaires faits par l'enquêté portent sur les éléments qu'il juge, lui, pour une raison ou pour une autre, importants. L'enquêteur mettant en œuvre un principe de relance minimum au moins au départ de cette deuxième phase, la longueur de l'entretien (environ 2 heures, ce qui est précisé dès le départ), le besoin presque constant des personnes de se justifier quant à leurs choix et non-choix, la situation même de face-à-face les conduisent à donner des éléments de justification qui dépassent très largement la simple description, rendue d'ailleurs inutile puisque le tableau effectue cette description de façon plus exhaustive et plus sûre que la personne.

La troisième phase aborde la question de la durabilité de façon plus frontale, dévoilant ainsi en grande partie l'objectif de ces entretiens et, plus largement, du programme de recherche, en ce qui concerne les pratiques de mobilités mais aussi les autres pratiques individuelles et collectives.

Le souci de cohérence de la personne se dévoilant, au moins en partie, dans le cadre d'un entretien, quels qu'en soient la forme et le degré d'ouverture, du très rapide micro-trottoir au récit de vie (et plus largement dans le cadre de toute situation relationnelle), renforce l'importance du cadrage que peuvent constituer, d'une part, le contexte de la situation et, d'autre part, la structure même du déroulé de l'entretien. C'est cette structure que nous proposons maintenant d'examiner en tant qu'elle permet d'amener - ou non - l'enquêté à donner tel type d'élément de réponse au questionnement du chercheur ou telle qualité de réponse. Cette structure est d'abord une structure temporelle dans la mesure où chaque élément d'information donné à entendre contribue à fixer un cadre pour l'enquêté dans lequel il se situe lorsqu'il poursuit l'entretien. Si la cohérence recherchée devait être parfaite, chaque instant $t$ de l'entretien déterminerait grandement chaque instant ultérieur. Cependant, il n'est pas tant recherché une cohérence parfaite qu'un certain niveau, acceptable, de cohérence. D'autre part, chaque phrase qui est dite échappe à celui qui la prononce, laissant du jeu dans la suite de l'entretien. Enfin, la personne peut anticiper sur ce qu'il va dire ensuite et par conséquent délivrer des éléments d'information en fonction de son objectif. Dans ce 


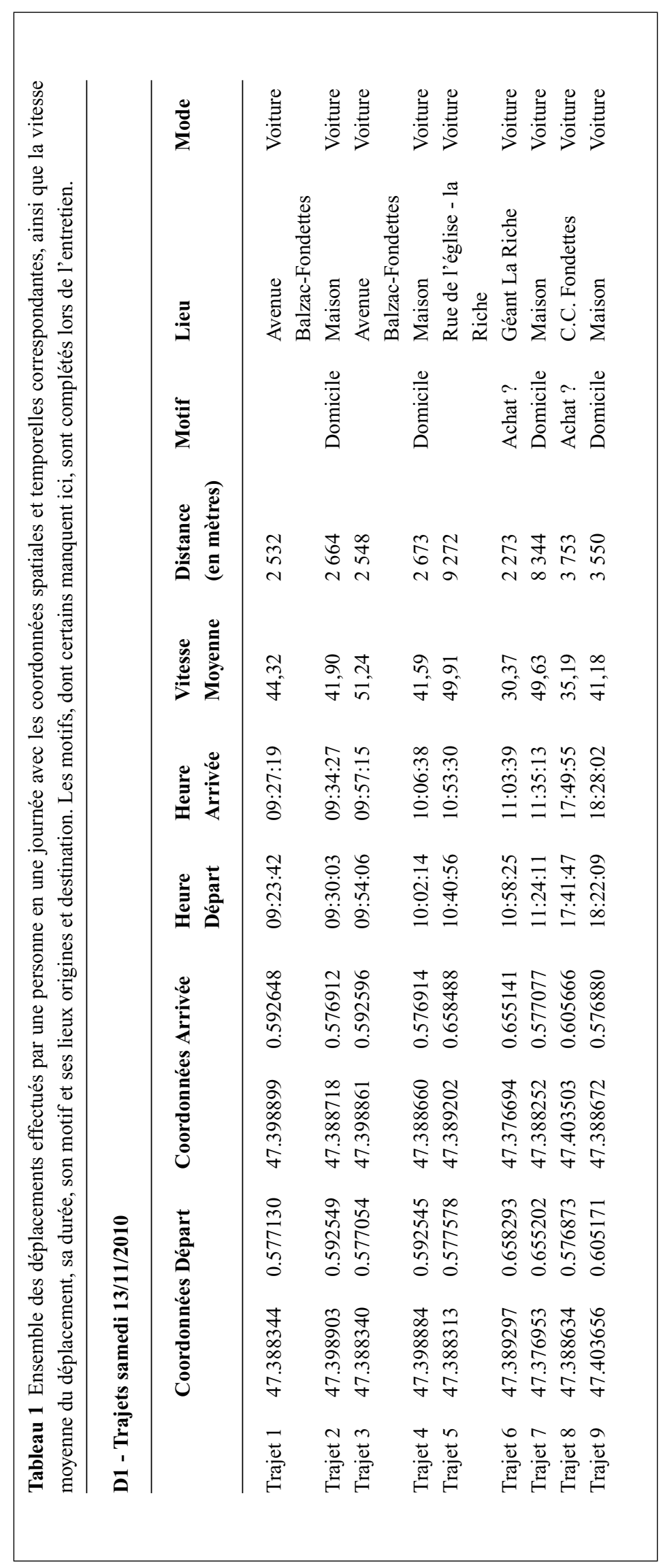

Necplus 
cas, ce sont les phrases qui suivent qui vont, par anticipation, déterminer ce qui est dit à l'instant $\mathrm{t}$.

Considérant cela, il est nécessaire d'accepter le fait que, dans la plupart des situations d'entretien, si c'est bien l'enquêteur qui fixe le cadre général qu'est la thématique de recherche plus ou moins précise et précisée à l'enquêté, c'est en revanche l'enquêté lui-même qui, par petites touches successives donne, en la créant, la cohérence d'ensemble, qui est une cohérence formelle, une cohérence d'image : image de soi vis-à-vis de l'autre et vis-à-vis de soi-même. Ainsi, les petits arrangements avec la réalité évoqués précédemment sont possibles, d'autant que l'enquêteur n'a généralement aucunement la possibilité de vérifier le rapport de vérité entre ce qui est dit et ce qui est fait. À une autre échelle ce cadrage s'effectue aussi en fonction des phases de l'entretien : ce n'est plus chaque élément d'information qui, par petites touches, influence ce qui suit, c'est chaque phase, formant un tout globalement cohérent qui prédétermine en partie les phases suivantes. Dans le cas du protocole présenté, il est possible de reconstruire ce phasage de la façon suivante : a) captation des données GPS ; b) entretien ouvert sur la mobilité générale de la personne ; c) confrontation de la personne à son tracé GPS qu'elle décrit et commente, justifiant par-là certains des aspects ; d) accentuation de la demande sur la durabilité des pratiques. La structure temporelle de ces quatre phases conduit au fait que la première phase cadre la deuxième, en partie puisque l'échelle temporelle des informations n'est pas la même : hebdomadaire pour la première, biographique pour la deuxième, le niveau de généralité est bien sûr très différent et la personne peut en jouer puisqu'elle n'a pas toujours porté un boîtier GPS lors de ses déplacements. De même, la deuxième fournit un cadre à la troisième et celle-ci à la quatrième, par une sorte d'effet d'entonnoir. Ce système permet d'évacuer en partie les biais évoqués précédemment et leurs conséquences ${ }^{9}$. Les mécanismes en œuvre peuvent être démontés.

Par rapport à un entretien classique, l'une des différences fondamentales provient du fait que la phase a (captation GPS) n'est pas une véritable situation de relation, en tout cas pas un face-à-face, et pourtant la personne donne déjà des informations. Cela signifie que c'est bien elle qui fournit le cadre mais dans un contexte qui ne lui permet pas de le choisir : les données GPS sont objectives et reconnues comme telles par l'enquêté lui-même. Ce faisant, toujours du fait de ce souci de cohérence, les éléments donnés par la suite ne pourront aller à l'encontre de ce qui a été enregistré de façon précise et exhaustive. On peut faire l'analogie avec un piège que la personne s'est tendu à elle-même,

\footnotetext{
${ }^{9}$ Il ne peut, bien évidemment, être question de quantifier ce gain de qualité puisqu'il est, dès le départ, difficile d'évaluer finement l'importance des conséquences de ces biais.
}

même si c'est sous l'injonction de l'enquêteur. Ainsi, les justifications données dans la troisième et la quatrième phase ne peuvent qu'être concordantes entre elles et avec ce qui précède.

Cependant, ce cadrage est effectif dès la deuxième phase, celle d'un rapide récit des mobilités : la personne sait même si elle ne se l'avoue pas nécessairement explicitement - que l'enquêteur sait, grâce au GPS, nombre d'informations la concernant ainsi que ses déplacements. Par conséquent, elle ne peut pas avoir des éléments discursifs qui seraient possiblement en contradiction flagrante avec les pratiques spatiales mesurées que pourtant elle connaît mais sur le mode du ressenti et celui du souvenir. Le fait même que l'enquêté ne sache pas exactement ce que sait l'enquêteur peut le rendre plus prudent quant à ce qu'il dit lors de cette première phase, cette prudence étant à entendre relativement à la liberté qu'il pourrait prendre vis-à-vis de la réalité de ses déplacements mais surtout la réalité de ses intentions en lien avec ces déplacements.

La troisième phase (celle de l'examen des tracés GPS) débute par le fait que la personne saisit pleinement et de façon explicite le niveau de précision de la mesure de ses déplacements et la nature des informations liées (déduites ou induites) à cette mesure, révélant ainsi son caractère véritablement inquisiteur ${ }^{10}$. La personne ne peut plus tricher avec les faits et le champ des justifications intellectuellement envisageables se réduit et se concentre sur les réelles motivations en lien avec les valeurs et les négociations internes à la personne - en œuvre ou non dans les délibérations préalables à chaque déplacement.

Quelques points d'analyse critique du protocole et de l'interprétation relative aux mécanismes en œuvre chez l'enquêté peuvent être avancés. Tout d'abord, cette interprétation repose sur le fait que la personne préfère donner une image cohérente de soi plutôt qu'une image valorisante, plus exactement que la valorisation par le fait de donner une image de cohérence de soi, même si celle-ci n'est que formelle, est plus forte que toute autre valorisation. Il n'a bien sûr pas été possible dans le cadre de l'enquête Périvia de conforter cette hypothèse.

D'autre part, l'analyse des tracés GPS n'implique pas nécessairement une seule possibilité de justification. Plusieurs peuvent s'agréger pour donner une idée précise de la motivation aux dimensions multiples qui a conduit

\footnotetext{
${ }^{10} \mathrm{Il}$ est possible de savoir, par exemple, si la personne, dans un train, était assise dans un wagon de queue ou un wagon de tête, si elle traverse les rues sur des passages protégés, si elle connaît bien le réseau de rues ou fait systématiquement des détours, etc. La personne peut même envisager la possibilité de savoir si elle dépasse les vitesses autorisées, si elle prend sa voiture pour faire à peine quelques centaines de mètres. Nos mesures n'ont pas été jusque-là mais la personne sent intuitivement qu'il est difficile de jouer avec l'enquêteur qui en sait possiblement plus sur elle qu'elle-même.
} 
à ce déplacement selon ces modalités particulières. Plus encore, un tracé ne permet pas de déduire une justification et une seule, il peut en induire une autre et l'enquêteur n'a pas les moyens d'une vérification. Mais l'enquêté ignore cela puisqu'il ne connaît pas l'étendue ni la précision des connaissances de l'enquêteur quant à ses déplacements ${ }^{11}$. Sa justification sera donc plus proche de la motivation réelle des modalités de son déplacement.

Enfin, il est extrêmement difficile d'extraire des éléments discursifs montrant ces contraintes puisque justement les informations données à entendre ont intégré ces contraintes. Repérer un tel élément discursif serait l'indice que la stratégie de l'enquêté de donner une image cohérente de soi n'a pas totalement abouti puisque, pour le repérer, il faut qu'il donne l'apparence d'une non-cohérence. Ceci dit, la cohérence n'est jamais totale.

\section{Conclusion}

L'individu ne peut pas ne pas prendre en considération son passé. Celui-ci agit sur lui, le fait agir. En revanche, l'individu dispose de moyens d'ordre psychologique pour que son passé ne s'impose pas intégralement à lui à tout moment : il est capable d'oubli, de la non-remémoration provisoire au refoulement le plus profond, mais aussi, parce que son cerveau n'est pas qu'une boîte cognitive dont la fonction première serait l'enregistrement de données, de souvenirs qu'il s'agirait de mobiliser au moment opportun, mais aussi une machine à raisonner couplée à une machine à émotion $[25,26]$, l'individu peut jouer, consciemment ou inconsciemment, avec son passé, donnant à chaque souvenir le composant une valeur, une importance relative, plus ou moins grande, voire quasi nulle. Ainsi, il fait avec son passé.

La situation d'enquête (comme nombre d'autres situations) rebat les cartes. Elle oblige à se remémorer sans qu'il soit possible de trier ce que l'on souhaite se remémorer et d'éviter ainsi de se ressouvenir de quelque élément passé. L'oubli est un effet essentiellement secondaire, au sens de Jon Elster [13]. La situation d'enquête conduit aussi à réévaluer ce que l'on a fait (ou pensé, ou dit...), y compris réévaluer l'évaluation faite dans le vif de l'action ou dans la préparation de celle-ci. La situation d'enquête montre la possibilité de s'être trompé sur ce qui a été fait, sur

\footnotetext{
${ }^{11}$ Le délai entre la captation des données GPS et le moment de l'entretien contribue à l'imprécision des souvenirs de la personne enquêtée et renforce le sentiment du décalage avec l'enquêteur qui, lui, a su mettre en image cartographique ces déplacements, en tableaux divers autres éléments de connaissance. L'enquêté suppose que l'enquêteur, par l'analyse, en sait plus que lui-même qui a seulement vécu ces déplacements pour d'autres raisons que la seule connaissance de les avoir fait.
}

l'évaluation de ce qui a été fait... sur soi, donc : d'une façon générale, elle présente à l'individu la possibilité de savoir combien il se trompe sur lui-même.

Le protocole que nous avons détaillé ne va pas à l'encontre de toutes ces difficultés. L'individu une fois enquêté doit toujours faire avec son passé et faire, dorénavant, avec le récit qu'il en a fait, mais cette technique permet de réduire le décalage entre ce qui a été et ce qui en est dit du fait du cadrage que permet et oblige à la fois le GPS et le caractère objectif des données qu'il apporte. On peut supposer alors que, par la réduction des biais évoqués et donc l'amoindrissement des conséquences de ceux-ci dans le recueil de matériau, relativement à une enquête classique fondée uniquement sur des entretiens, le récit est, sinon plus véridique, du moins plus sincère.

\section{Bibliographie}

1. Bourdieu P (1993) La misère du monde. Seuil.

2. Chardonnel S (2011) Projet TRACES : évaluation comparée de l'apport de l'assistance GPS aux enquêtes de mobilité. communication au séminaire Urban Fabrik, Grenoble, 7 décembre 2011.

3. Depeau S (2011) À la recherche des espaces invisibles de la mobilité : usage, apports et limites des techniques GPS dans l'étude des déplacements urbains à l'échelle pédestre. Communication au séminaire Urban Fabrik, Grenoble, 7 décembre 2011.

4. Énaux C (2006) ItiRestitution : analyse spatio-temporelle des données GPS pour la restitution des parcours et des arrêts du déplacement. http://halshs.archives-ouvertes.fr/halshs-00563661/en/.

5. Feildel B (2011) Perspectives pour le ménagement des espaces de vie périurbains : un éclairage par les relevés GPS et l'herméneutique cartographique. Communication au séminaire Urban Fabrik, Grenoble, 7 décembre 2011.

6. Martouzet D (2012) Durabilité des territoires périurbains et attitudes des habitants face à la notion de durabilité. Puca.

7. Lévi-Strauss C (2001) Tristes tropiques. Seuil.

8. Chalas Y (2000) L'invention de la ville. Anthropos.

9. Neuman WL (1991). Social research methods: Qualitative and quantitative approaches. Allyn and Bacon.

10. Sudman S, Bradburn NM (1974) Response effects in surveys. Aldine.

11. Saint Augustin (1964) Les confessions. Garnier-Flammarion.

12. Freud S (2010) Cinq leçons de psychanalyse. Payot.

13. Elster J (1986) Le laboureur et ses enfants. 2 essais sur les limites de la rationalité. Éditions de Minuit.

14. Nozick R (1969) Newcomb's Problem and Two Principles of Choice. In: Rescher N (eds) Essays in Honor of Carl G. Hempel. D Reidel, pp. 114-146.

15. Davidson D (1991) Paradoxes de l'irrationalité. Éditions de l'éclat.

16. Sartre JP (2008) L'être et le néant. Essai d'ontologie phénoménologique. Gallimard.

17. Dupuy JP (1994) Temps et rationalité. Cahiers d'économie politique 24-25 : 69-104.

18. Engel (1991) Avant-propos. In: Davidson D Paradoxes de l'irrationalité. Édition de l'éclat, pp. 9-18.

19. Bourdieu P, Passeron JC (1970) La reproduction, éléments pour une théorie du système d'enseignement. Édition de Minuit. 
20. Martouzet D, Bailleul H, Feildel B (2011) Les justifications de la mobilité périurbaine : mise à l'épreuve de la durabilité dans un espace intermédiaire. In: Giroud M, Mainet H, Édouard JC (eds) Les mobilités spatiales dans les villes intermédiaires, Presses Universitaires de Clermont-Ferrand, pp. 11-30.

21. Thibault S, Bailleul H, Martouzet D, Feildel B (2011). La fabrique d'un habiter périurbain : le transport et la centralité. Les Cahiers de l'Association de Prospective Rhénane 2 : 41-63.

22. Feildel B, Martouzet D (2012) La mobilité comme modalité de l'ancrage : enrichir l'évaluation de la durabilité des espaces périurbains. Recherche Transports Sécurité 110-111: 1-19.
23. Martouzet et al. (2012) Le périurbain à l'épreuve des modèles d'habiter, la viabilité périurbaine entre théorie(s) et pratique(s). CITERES/Puca.

24. Thévenot L (1996) Justifications et compromis. In: Canto-Sperber M (éd.) Dictionnaire d'éthique et de philosophie morale, PUF, pp. 789-794.

25. Damasio A (1995) L'erreur de Descartes : la raison des émotions, Odile Jacob.

26. Damasio A (2005) Spinoza avait raison : joie et tristesse, le cerveau des émotions. Odile Jacob. 